This is the final peer-reviewed accepted manuscript of:

R. Meattini, S. Benatti, U. Scarcia, D. De Gregorio, L. Benini and C. Melchiorri, "An sEMG-Based Human-Robot Interface for Robotic Hands Using Machine Learning and Synergies," in IEEE Transactions on Components, Packaging and Manufacturing Technology, vol. 8, no. 7, pp. 1149-1158, July 2018.

The final published version is available online at DOI: 10.1109/TCPMT.2018.2799987

Rights / License:

The terms and conditions for the reuse of this version of the manuscript are specified in the publishing policy. For all terms of use and more information see the publisher's website.

This item was downloaded from IRIS Università di Bologna (https://cris.unibo.it/)

When citing, please refer to the published version. 


\title{
A sEMG-Based Human-Robot Interface for Robotic Hands Using Machine Learning and Synergies
}

\author{
R. Meattini, S. Benatti, U. Scarcia, D. De Gregorio, L. Benini and C. Melchiorri
}

\begin{abstract}
Developing natural control strategies represents an intriguing challenge in the design of Human-Robot Interface (HRI) systems. The teleoperation of robotic grasping devices, especially in industrial, rescue and aerospace applications, is mostly based on non-intuitive approaches, such as remote controllers. On the other hand, recent research efforts target solutions that mimic the human ability to manage multi-finger grasps and finely modulate grasp impedance. Since electromyography (EMG) contains information about human motion control, it is possible to leverage such neuromuscular knowledge to teleoperate robotic hands for grasping tasks. In this article we present a HRI system based on 8 fully-differential EMG sensors connected to a wearable sensor node for acquisition and processing.

By virtue of a novel bio-inspired approach, the embedded myocontroller merges pattern recognition and factorization techniques to combine a natural selection of the robotic hand configuration with the proportional control of the related grasps. The HRI system has been fully designed, implemented and tested on two robotic hands: a dexterous anthropomorphic hand and a three-fingered industrial gripper mounted on a robotic manipulator. Results of the test performed on 4 able-bodied subjects show success rates greater than $90 \%$ reached in grasping objects that require different hand shapes and impedance regulations for the task completion. The outcomes also show that the users modulate the bio-inspired degrees of control in a natural manner, proving the pertinence of the proposed system for an effective human-like control of robotic grasping devices in a wearable form-factor.
\end{abstract}

Index Terms-Embedded Systems, Human-Robot Interface, EMG Sensors, Robotic Hands, Industrial Gripper, Grasping, Pattern Recognition, NMF.

\section{INTRODUCTION}

$\mathbf{R}$ OBOTICS is currently facing a new generation of challenges characterized by a growing demand for a closer cooperation and interaction with humans. In recent years, this is leading the design of robotic systems themselves towards human-inspired solutions, as a road to replicate the human ability and flexibility in performing motor tasks. For this purpose, one key element is the so-called human-in-the-loop integration, where the human interaction is an inherent element of the system design, in the overall framework of HRIs.

A major challenge to enable a natural interaction between humans and robots is the leverage of neural signals acquired from the human body to communicate with the machines. Despite recent progresses of Brain-Computer Interfaces [1], [2] in several scenarios ranging from rehabilitation [3] to robotic control [4], systems based on EMG signals are the most popular approaches for the control of robotic hands [5], [6]. Indeed, although this technique has not reached an industrial maturity yet, such a trend reflects the goodness of noninvasively recordings, long-term stability and ethical constraints of

The authors are with the Dept. of Electrical, Electronic and Information Eng., Univ. of Bologna, Italy; e-mail: \{roberto.meattini2, simone.benatti, umberto.scarcia, luca.benini, claudio.melchiorri\}@unibo.it. L. Benini is also with the Dept. of Information Technology and Electrical Eng., ETH Zurich, $\mathrm{CH}$, e-mail: luca.benini@iis.ee.ethz.ch.

This research has been partially supported by the University of Bologna, with the FARB Linea 2 funding action and from Micropower deep learning SNF project and EuroCPS EU projects.
EMG measurements [7]. The aim of a myoelectric(ME)-driven control system consists on the online extraction of control signals from EMG measurements to regulate the behavior of external devices through specific commands [8]. Furthermore, it has been demonstrated that human motion intentions can be detected with good accuracy from surface electrodes EMG (sEMG) signals [9]. This because muscle biopotential contains information about neural drives (e.g. spike trains of motoneurons) and, consequently, on motor tasks [10].

However, despite the inherent potential of sEMG-based interfaces, current ME HRIs for grasping tasks only partially fulfil user-centered requirements, which include the need for an increased number of functions, lower reaction/execution times and intuitiveness of the control systems. In particular, to improve users acceptance, the development of more intuitive control interfaces is the most influential necessity [11]. In fact, several applications in teleoperation and higher level telesupervision of robotic grasping devices could benefit from human-inspired controlled ME HRIs, as astronaut commanding of construction robots in space missions, operators of disaster search and rescue robots, direct operations of bomb disposal experts and dexterous hand prosthetic rehabilitation [12], [13]. To this end, the consideration of the overall hand musculoskeletal structure in the HRI design process can result a fundamental point. The human hand is a very complex and versatile sensorimotor system characterized by 21 degrees of freedom (DoF) controlled by 29 muscles [14]. This multitude of DoF can be dimensionally reduced thanks to the concept of postural synergies [15], according to which the grasp synthesis for a large set of objects is possible by linearly combining only a few dominant hand postures (i.e. postural synergies or eigenpostures). In addition, recent comprehensive surveys have highlighted how the overall synergistic organization underlying motor control of the human hand reflects a spatial and temporal coordination at different levels: postural, muscular and neural [16]. In this connection, it is significant that linear decomposition algorithms such as Non-negative Matrix Factorization (NMF) or Independent Component Analysis (ICA) have been used to extract muscular synergistic weights, which represent the modulation of supraspinal activation signals [17], [18]. This is in agreement with the concept of muscular synergies, according to which multiple muscles can be activated as a unit by a single neural drive [19]. Moreover, another fundamental aspect of the human hand control is the modulation of the grasp stiffness in order to accomplish a wide number of grasping tasks, thanks to antagonistic muscles coactivations that allow the regulation of joints impedance [20], which defines the dynamic relation between force and velocity in a joint [21].

In this work we present a sEMG-based HRI for human-like control of robotic hands for grasping tasks. We designed a wearable sensor interface which implements a ME hybrid control system, exploiting machine learning (ML) for the selection of an appropriate grasp hand shape and factorization to extract 


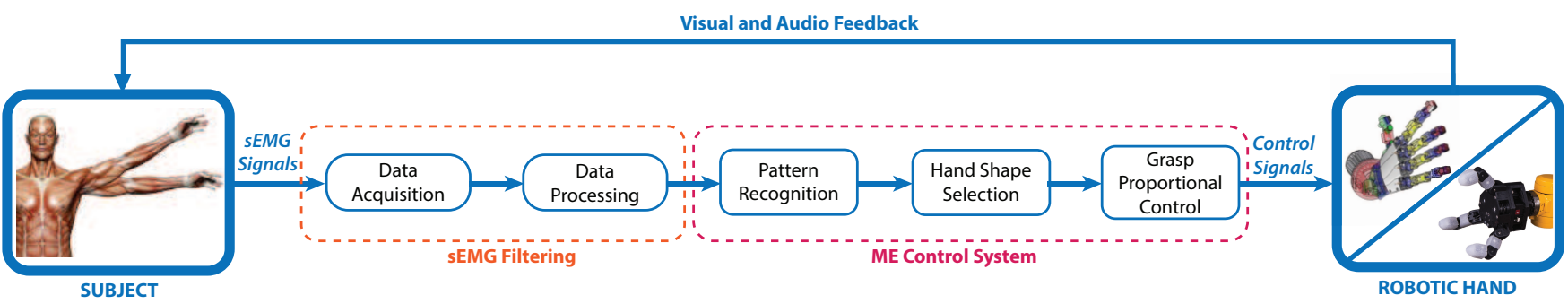

Fig. 1: Scheme of the sEMG-based HRI system.

supraspinal neural drives related to the user hand grasp control. These neural drives, online derived from sEMG signals, are used to replicate on the robotic hand two human-like features: continuous closure of a selected grasp configuration and a variable stiffness control. In fact, our aim is to show that combining ML and factorizarion based control approaches, it is possible to realize a HRI to naturally control a robotic hand, involving also postural and muscular synergy concepts in the control system synthesis process. The HRI has been tested in several grasping tasks controlling the UB Hand IV (University of Bologna Hand, version IV) anthropomorphic robotic hand [22], [23], and a three-fingered 3D printed robotic gripper for industrial applications.

The paper is organized as follows. In Section II the related work is reported. Section III presents the hardware tools. The details of the developed HRI are described in Section IV and the results of the experimental tests are shown in Section V. Finally, Sec. VI draws the conclusions.

\section{RELATED WORK}

In the research literature, different approaches have been proposed for the implementation of HRIs for the control of robotic hands based on forearm's muscles sEMG signals. A number of studies have focused on ME control systems that make use of two sEMG channels, placed normally on an antagonistic muscle pair. In such methods the user switches between different functions mainly by means of muscles co-activations, then the related robotic hand $\mathrm{DoF}$ are actuated proportionally to muscle activity values once the amplitude of a processed sEMG channel exceed a proper threshold. Several commercial prosthesis are controlled in this manner, [10], such as the Ottobock Michelangelo Prosthetic Hand [24], the BeBionic hand [25] or the Touch Bionics i-Limb Hand [26]. In general, commercial and clinical ME-based HRIs for robotic hands have reached a considerable level of functionality. However, their control systems do not take advantage of improvements in this field by research laboratories [11].

To deal with the various configurations assumed by the human hand during grasping tasks, novel views have been proposed, based on the mapping between forearm sEMG signals and hand postures using ML techniques [27], e.g. [28], [29]. In particular, pattern recognition based ML approaches have recently been employed extensively [30], achieving extremely high performance (i.e. more than $95 \%$ classification accuracy) [31], [32]. However, mainly because of reliability issues [33], none of the ML-based systems proposed so far has been implemented in real applications, whether clinical, commercial or generic telemanipulation systems are considered. In fact, ML approaches suffer particularly from ambiguity when the input signal crosses two decision zones, and from factors as electrode positioning, sweat and muscle fatigue [16]. Thus, in the last years regression and signal factorization methods are gaining ground in the research community as approaches to deal with the reliability issue of ML-based ME control systems [16].

Regression and factorization algorithms are at the base of simultaneous and proportional DoF control systems [34], in which multi-muscle EMG signals are mapped onto continuous DoF movements. The advantage of such a method lies in the possibility of continuously controlling the DoF, so that ambiguities in input signals do not lead to totally unwanted results. However, despite several positive outcomes, also regression and factorization-based ME HRIs have not been implemented yet in real systems. This is probably due to certain assumptions on which the method is based, consisting in small crosstalk between sEMG channels and linearity between muscle's activation functions and sEMG signals [34]. These conditions are not satisfied for human hand's muscles, resulting in degraded performance and instability when more than two DoF are considered [34].

In this work, we address these problems proposing a complementary view of the two approaches, combining their positive aspects: ( $i$ ) the ML-based approach gives the possibility to actuate multiple DoF configurations, and therefore it is used for the selection of only shapes of the grasp, overcoming a discrete behaviour in the grasp opening/closure that can lead to catastrophic results (e.g., the robotic hand opens while grasping a glass); (ii) the regression-based approach provides the capability to continuously control DoF, and therefore it is used to regulate the closure and the stiffness by means of a unique coordinated motion (the overall opening and closing of the users hand), overcoming the critical deterioration in performance when more than two DoF are proportionally controlled (that we avoid providing the multiplicity of the grasp shapes by means of the ML approach).

For these reasons, in this paper we propose a hybrid ME HRI including both ML and factorization techniques, in a unique control framework that also exploits the human hand neuromuscular knowledge introduced in Section I.

\section{EXPERIMENTAL TOOLS AND SETUP}

A general scheme of the developed HRI is shown in Fig. 1. sEMG signals acquired from the user forearm are processed to extract information used for two purposes: $(i)$ the selection of the grasp hand shape by means of gesture recognition via a Finite State Machine (FSM) logic, (ii) a proportional control strategy for the closure through the stiffness control of the selected grasp. Two feedback signals are available for the user: a visual feedback, due to the observation of the device and the task evolution, and an audio feedback, that helps the user to understand the entity of his/her action on the device and therefore to manage it in a more effective and stable way. Two different robotic hands have been used: the anthropomorphic UB Hand IV and a three-fingered gripper. Furthermore, a 


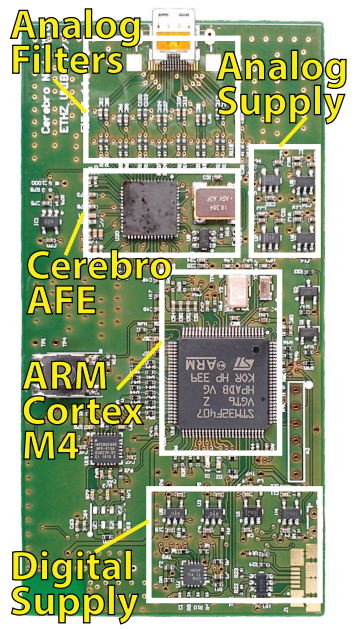

(a)

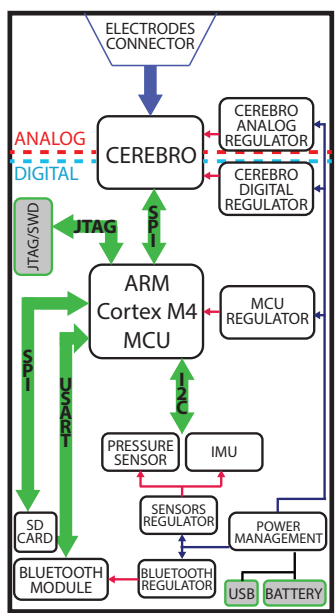

(b)
Fig. 2: Wearable sensor node, (a) Architecture diagram (b) populated board

graphically simulated three-fingered gripper has been used for an initial training phase.

\section{A. sEMG Setup}

The wearable sensor node used in this application is designed on a 6-layers printed circuit board (PCB). Fig. 2(a) shows a picture of the board, while the architectural diagram of the embedded node is depicted in Fig. 2(b). The node [35] is designed for the acquisition of analog bio-signals in wearable multisensory applications through Cerebro, a highperformance Analog Front End (AFE) [36] connected via SPI to an ARM Cortex M4 Microcontroller. Data are acquired at $1 \mathrm{kHz}$ and streamed to a PC via a standard 2.0 Bluetooth interface. The board is battery-powered and is also equipped with an IMU interface and a pressure sensor that can be used for future improvements of the HRI. Since the analog signal acquisition is a critical task, it requires careful design of board layout and component placement to minimize noise and interference on the signal acquisition. In this board we separated analog and digital circuitry, providing dedicated low drop out (LDO) regulators for the microcontroller and for the AFE as shown in Fig. 2(b). In this application, 8 sEMG channels (i.e. 8 pairs of electrodes in fully differential configuration) are acquired from the user's forearm muscles. Low-cost disposable surface skin electrodes equipped with conductive gel are used. The electrodes are equally distributed around the forearm forming an armband, as shown in Fig. 3(a), 3(b). The sEMG armband is positioned to provide information both on hand gestures and grasp closure, as described in Sect. IV-A and IV-B. Therefore, it is placed on the Flexor Digitorum Superficialis and the Extensor Digitorum Communis muscles, because these muscles are involved in digits flexion and extension, referring to methods and best practices outlined in [37], Fig. 3(a).

By virtue of robust algorithms used for the $\mathrm{ME}$ control strategies and of the proper sensor coverage of the forearm, the system reaches recognition accuracy higher than $90 \%$ as proved in Sect. V, even if electrodes are slightly misaligned with respect to the exact muscle position. A filtering procedure

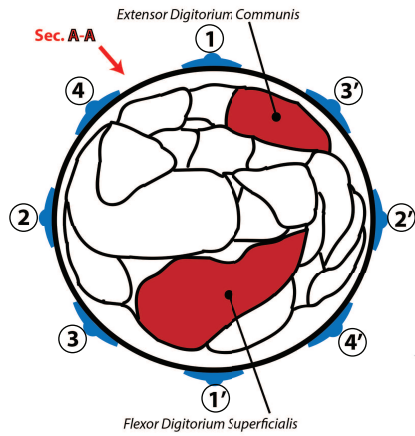

(a) Forearm section.

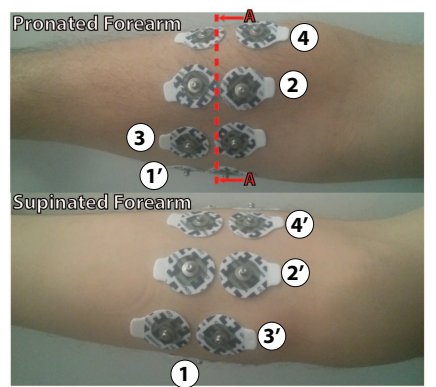

(b) Pronated and supinated forearm electrodes placement views.
Fig. 3: Electrodes placement and gestures to be recognized by the SVM classifier.

is implemented for each channel of the sEMG data. The processing chain consists of: $(i)$ a $50 \mathrm{~Hz}$ notch filter for powerline interference cancellation; ( ii) a $20 \mathrm{~Hz}$ band-pass filter, that results in the best compromise to reduce baseline noise (mainly thermal, chemical and movement artefact noises) and obtain the desired information content [38], [39]; and finally (iii) the root mean square (RMS) value of the signal calculated on a $200 \mathrm{~ms}$ window with no overlapping.

\section{B. Robotic hands: control architecture and device description}

Two robotic devices have been used to test the ME control system: the UB Hand IV [22], [23], as anthropomorphic device (to perform human-like grasping tasks), and a three-fingered gripper mounted on a 6 DoF industrial manipulator (to perform teleoperation in a manufacturing simulated application). Note that the two devices have different kinematics, number of joints and number of DoF. However, both robotic hands are actuated by tendons driven by Dynamixel servomotors and therefore they have the same control architecture.

1) Controller architecture: The control architecture is schematically shown in Fig. 4. Let consider, for both the hand and the gripper, $n_{J}$ the total number of joints of all fingers, and $n_{m}$ the number of tendons actuating the joints, each one driven by a servomotor. The architecture implemented to control both joints position and the grasp stiffness has a threelevel hierarchical structure: motor, joint and synergy control level.

At the lower level the Dynamixel servomotors, thanks to their embedded compliance [40], are controlled according to

$$
\tau_{m}(t)=K_{m}\left(\theta_{m}^{r e f}(t)-\theta_{m}(t)\right)
$$

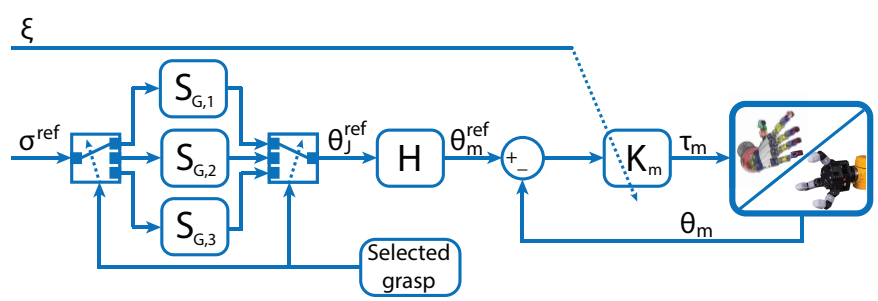

Fig. 4: Robotic hands controller architecture. 
where $\tau_{m} \in \mathbb{R}^{n_{m}}$ is the motor torques vector, $K_{m} \in \mathbb{R}^{n_{m} \times n_{m}}$ is a diagonal matrix with the desired motor stiffness values, $\theta_{m}^{r e f}, \theta_{m} \in \mathbb{R}^{n_{m}}$ are respectively the reference and the actual position of the motors. To move to the joint control level, a mapping $H \in \mathbb{R}^{n_{m} \times n_{J}}$ between the joint and motor spaces is necessary (for more details see [22], [23]). Using this mapping, the reference angle of the motors can be computed once the desired joint configuration $\theta_{J}^{r e f}$ is known, that is

$$
\theta_{m}^{r e f}(t)=H \theta_{J}^{r e f}(t) .
$$

Using (2), (1) can be mapped in the joint space as

$$
\tau_{J}(t)=K_{J}\left(\theta_{J}^{r e f}(t)-\theta_{J}(t)\right),
$$

where the joint stiffness matrix is computed as $K_{J}=H^{T} K_{m} H \in \mathbb{R}^{n_{J} \times n_{J}}$.

The definition of the joint reference configuration $\theta_{J}^{\text {ref }}$ is given at the higher synergy control level, computed as

$$
\theta_{J}^{r e f}(t)=S_{G, i} \sigma^{r e f}(t), \quad i=1,2, \ldots, n_{g},
$$

where $\sigma^{r e f}$ is the synergistic hand closure reference given by the ME control system (see (9)), $S_{G, i} \in \mathbb{R}^{n_{J} \times 1}$ is referred to as the grasp synergy matrix and $n_{g}$ is the number of grasp shapes that the robotic hand is programmed to perform, based on the application. In our HRI system we have $n_{g}=3$ and therefore $S_{G, 1}, S_{G, 2}$ and $S_{G, 3}$ represent the specific postural patterns of the grasps G1, G2 and G3, reported in Tab. I. The last two columns of Tab. I are referred to the user's gestures not correlated with grasping, i.e. the open hand and the neutral pose. Such gestures are used in the control FSM and described below (see Sec. IV-A1) .

Besides the position control, the modulation of the grasp stiffness can be achieved by acting on $K_{m}$, since it results that $K_{J}=H^{T} K_{m} H$. Thus, the stiffness gains in $K_{m}$ are modulated as

$$
K_{m}=K_{m}(t)=\operatorname{diag}\left\{k_{\min }+\left(k_{\max }-k_{\min }\right) \xi(t)\right\},
$$

where $k_{\min }$ and $k_{\max }$ are the minimum/maximum settable stiffness values, and $\xi(t)$ is the grasp stiffness level determined according to the ME control of the grasp, see (9).

Therefore, with the control architecture outlined so far, it is possible to regulate both grasp closure and stiffness of the robotic hand.

2) UB Hand IV: The UB Hand IV is a dexterous anthropomorphic robotic hand [22], [23], Fig. 5(a). The hand is fully actuated with a particular actuation system, requiring for each finger of the hand (with 3 actuated DoF) 5 coupled tendons. Then, referring to the control architecture of Sect. III-B1, there are $n_{j}=15$ DoF actuated by $n_{m}=25$ tendons, each one driven by a Dynamixel RX-24F servomotor [40].

In particular, (4) is implemented on the UB Hand IV in order to realize the hand shapes related to the Power, Tripodal and

TABLE I: Gestures to be classified and robotic hand grasps.

\begin{tabular}{ccccc}
\hline $\begin{array}{c}\text { User's } \\
\text { gesture }\end{array}$ & $\begin{array}{c}\text { Gesture } \\
\text { label }\end{array}$ & $\begin{array}{c}\text { UB Hand IV } \\
\text { grasp }\end{array}$ & $\begin{array}{c}\text { Three fingered } \\
\text { gripper grasp }\end{array}$ & $\begin{array}{c}\text { Grasp } \\
\text { label }\end{array}$ \\
\hline Three Fingers & TF & Tripodal grasp & Tripodal grasp & G1 \\
\hline Ulnar Pinch & UP & Ulnar grasp & Parallel grasp & G2 \\
\hline Fist & F & Power grasp & Cylindrical grasp & G3 \\
\hline Open Hand & OH & - & - & - \\
\hline Neutral Pose & NP & - & - & - \\
\hline
\end{tabular}

Ulnar grasps. In particular, the weights of the grasp synergy matrices $S_{G, 1}, S_{G, 2}$ and $S_{G, 3}$ are determined by modifying the first postural synergy matrix, implemented on the UB Hand as in [41], such that the hand pose given by (4) for the maximal value of $\sigma^{r e f}(t)$ matches the hand shape of the respective grasp of interest. The UB Hand joint configurations, obtained by varying the value of the synergistic hand closure reference $\sigma^{r e f}(t)$, are shown in Fig. 5(a).

3) Three fingered gripper: The gripper used in this work has three fingers and a palm, see Fig. 5(b). Each finger is composed by three phalanges. Two fingers have 3 joints each: one for the proximal phalanx adduction/abduction movement $(\mathrm{Ad} / \mathrm{Ab}$ joint) and the other two for the middle and distal phalanges flexion/extension motions. The third finger has only 2 articulations (the $\mathrm{Ad} / \mathrm{Ab}$ joint is not present). Therefore, the total number of joints is 8 . However, the gripper is underactuated by means of a specific actuation system, where, according to the notation of the controller architecture in Sect. III-B1, $n_{m}=3$ Dynamixel RX-106T servomotors [40] actuate $n_{J}=3$ DoF by means of a proper pulley-based tendon network. The 3 controlled DoF are: the abduction/adduction motion of the two $\mathrm{Ad} / \mathrm{Ab}$ joints, and, independently, the flexion/extension motion of the middle and distal phalanges.

The three grasps controlled by the ME control system for the gripper are the cylindrical, tripodal and parallel grasps. Specifically, referring to Fig. 5(b), the weights of the related grasp synergy matrices $S_{G, i}$ are set empirically in order to have a suitable coordinated flexion of the hand's phalanxes for the cylindrical grasp. The synergy matrix is then modified to have the $\mathrm{Ad} / \mathrm{Ab}$ joint angles equal to $\pi / 6 \mathrm{rad}$ for the tripodal grasp and the distal joint angles equal to the opposite of the proximal joint angles value for the parallel grasp.

\section{Methods And Procedures}

\section{A. Grasp hand shape selection}

The classification of the hand gestures for the different types of grasps is implemented by means of the Support Vector Machine (SVM) pattern recognition technique [42], [43]. The SVM is a supervised ML algorithm in which the training is based on the solution of a convex optimization problem to find the optimal separation hyperplane between two classes of a trainset. The outcome of the training algorithm is then used by the classifier to discriminate between the two classes in new data. The procedure can be applied to $n$-class problems

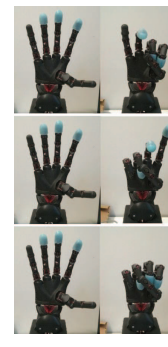

(a) Top to Ulnar and Power grasps. bottom: Tripodal,

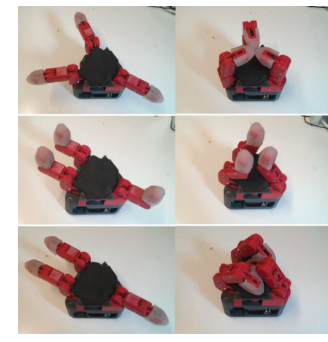

(b) Top to bottom: Tripodal, Parallel and Cylindrical grasps.

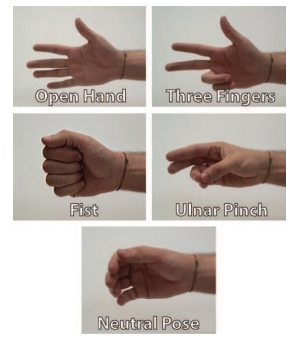

(c) Gestures to be classified.
Fig. 5: UB Hand IV (a), gripper (b) and hand gestures for the selection of the grasp hand shape (c). 


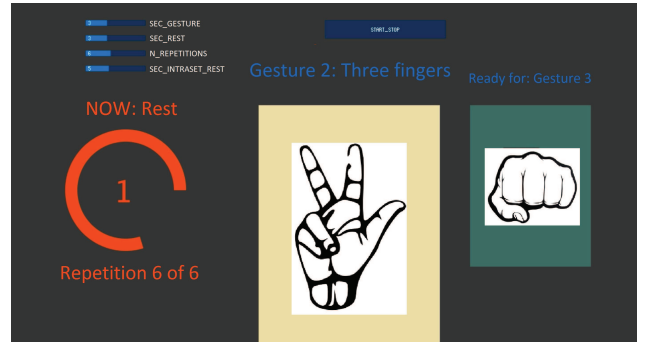

Fig. 6: Graphical interface for the SVM training dataset.

by means of a One Versus One approach. In this work the SVM classifier is implemented using the libSVM opensource library [44] for Matlab.

1) SVM training protocol: In order to build the classifier, a training session is required to collect the training dataset, that is composed by 8-dimensional samples of the RMS values of the sEMG channels (see Subsection III-A and Subsection V-A). Thereafter, the SVM classifier can be used online to recognize hand gestures. The five gestures to be classified are shown in Fig. 5(c) and reported in Tab. I: Open Hand, Three Fingers pose, Fist, Ulnar Pinch, and Neutral Pose. The two gestures not associated to any shape (Open Hand and Neutral Pose) are used according to a grasp transition logic (see below). The training dataset to be acquired consists in the repetition of each gesture (without considering the Neutral Pose) 6 times. Every gesture has to be executed for 3 seconds followed by 3 seconds where the user has to rest his fingers (Neutral Pose). The Neutral Pose duration become 6 seconds between two different gesture repetition groups. Note that the execution of 24 gestures with a precise timing is a not trivial operation. This can be tedious and difficult, and may affect user's motivation and therefore the HRI performances. For this reason a graphical interface that helps the user in executing of the sequence of gestures has been developed, see Fig. 6. This interface shows on a screen a timer, with different colours for gesture execution and finger rest moments, and displays which gesture has to be performed, the number of the current repetition, and advises in advance when a new group of gestures has to be performed.

2) FSM grasp hand shape transition logic: In order to exploit the pattern recognition for the online selection of the grasp hand shape, a FSM logic has been implemented, see the

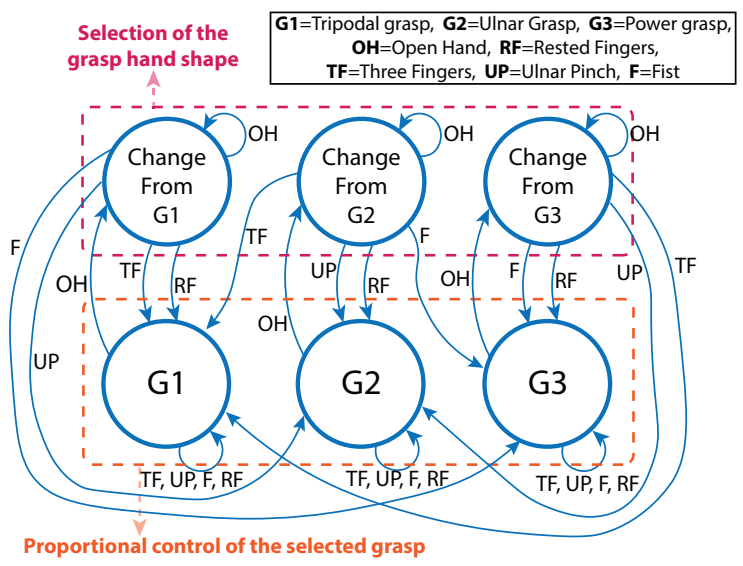

Fig. 7: FSM for the hand shape transition logic.

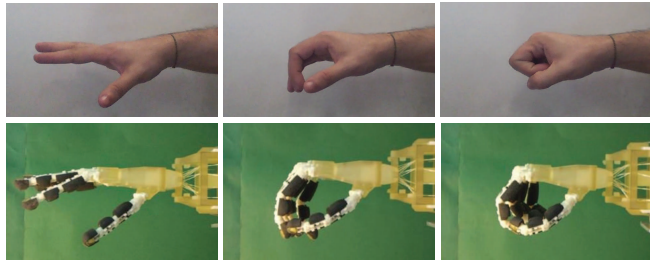

Fig. 8: Comparison between user's and robotic hand motion with the first postural synergy.

graph of Fig. 7. The Open Hand is an exit gesture that the user has to perform in order to access to the grasp selection states, in which the system waits for a new shape indication. If a new gesture is recognized by the SVM classifier, a new grasp shape is enabled. Once the Open Hand gesture has been detected, the user can also execute the Neutral Pose to remain in the current configuration. When the user enters in a grasp control state of the FSM, i.e. G1, G2 or G3 in Fig. 7, the robotic hand takes the joint configuration corresponding to the selected shape. As an exemplifying case, if the current state is G1, to access the grasp control state G2 the user has to perform Open Hand followed by Three Fingers, whereas, to access G3, has to perform Open Hand followed by Fist. Thereafter, once a grasp control state is activated, the proportional control can be used to regulate closure and stiffness of the selected grasp, see the following Sect. IV-B.

\section{B. sEMG proportional control of the grasp}

The concepts of muscular and postural synergies have been jointly used in the HRI system to provide the user with a natural proportional grasp control of the robotic hand. In humans, on the basis of the muscular synergy concept, a unique neural drive is shared by different muscles, individually activated with a certain degree of excitation determined by spinal cord circuitries (i.e., the synergy weights). The application of the MNF algorithm ${ }^{1}$ on the multichannel sEMG signal extracts the values of such muscular sinergies.

In this work, the NMF algorithm is applied to forearm's sEMG signals to determine the muscular synergy matrix related to hand motions, in order to implement a myoelectric proportional control of the grasp. Since the proportional control approach presents instability when more than two DoF are considered (see Section II), we determine muscular synergies for only one postural synergy-based DoF: the coordinated opening and closing movements of the user's hand fingers (see on the top of Fig. 8), in order to regulate both closure and stiffness of the robotic hand during grasping tasks.

Thus, the grasp hand shape is firstly selected by the SVM classifier as illustrated in the previous subsection, whereas all the grasps are controlled by the same overall opening and closing hand movement.

1) Extraction of muscular synergies for grasp control: Let us consider the RMS value of a 8-channel sEMG recording (see Sect. III-A), corresponding to $n$ samples and collected in the matrix $E \in \mathbb{R}^{8 \times n}$. On the basis of the sEMG generative model proposed in [34], $E$ can be expressed as

$$
E=S_{M} U
$$

${ }^{1}$ Given a nonnegative matrix $A \in \mathbb{R}^{m \times n}$ (a matrix whose elements are all non negative), the product $W H$ is called NMF of $A$ if nonnegative matrices $W \in \mathbb{R}^{m \times k}$ and $H \in \mathbb{R}^{k \times n}$, with $k<\min (m, n)$, are found such that the functional $f(W, H)=\frac{1}{2}\|A-W H\|_{F}^{2}$ is minimized [45]. 


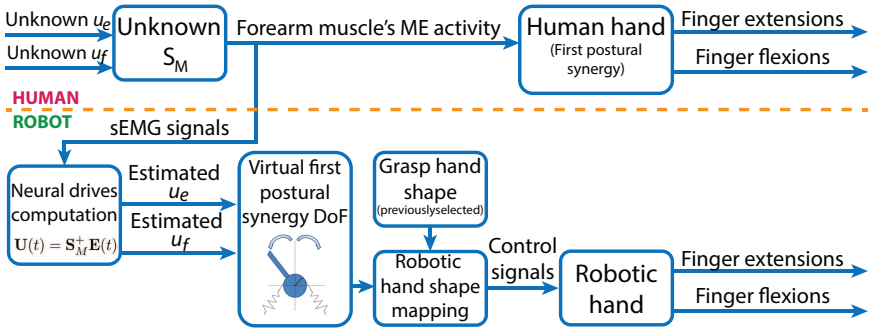

Fig. 9: Conceptual block diagram of the HRI control architecture from a synergy-centered point of view.

where $S_{M} \in \mathbb{R}^{8 \times 2 d}$ is the muscular synergy matrix and $U \in \mathbb{R}^{2 d \times n}$ is the neural drive matrix, with $d$ the number of DoF controlled during the sEMG acquisition. In (6), $U$ and $S_{M}$ are unknown and can be assumed nonnegative, since they represent the values of $2 d$ neural drives modulated by $2 d \cdot 8$ muscular synergy weights, respectively. Therefore, the activation of each DoF can be expressed in terms of two nonnegative control signals of $U$. In our case, the overall opening and closing motion of the hand fingers is assumed to be the only controlled DoF $(d=1)$. It follows that the matrix $E$ represents the muscle activity during the regulation of such a single DoF. Furthermore, it is worth to note how such movement involves all the joints of the hand and the wrist, see Fig. 8, and can be reasonably associated with the hand motion when only the first eingenposture of postural synergies is considered. According to this, the concept of the antagonistic actuation model [46] for the mentioned synergistic DoF is exploited.

We consider two antagonistic actions, representing two groups of forearm flexor and extensor hand muscles, that control the synergistic DoF reference and the stiffness level of the hand. In this relation, it is possible to consider two neural drives that activate such antagonistic actions. Hence, the matrices $S_{M}$ and $U$ in (6) can be written for only one DoF, that is $d=1$, as

$$
S_{M}=\left[s_{M_{e}} s_{M_{f}}\right], \quad U=\left[\begin{array}{l}
u_{e}^{T} \\
u_{f}^{T}
\end{array}\right]
$$

where $s_{M_{e}}, s_{M_{f}} \in \mathbb{R}^{8 \times 1}$ are the extension and flexion components of the muscular synergy matrix and $u_{e}, u_{f} \in \mathbb{R}^{n \times 1}$ are the extension and flexion components of the neural drive matrix. Therefore, taking into account (7), there is only one possible solutions of (6) with respect to $S_{M}$ and $U$, that can be computed by the NMF algorithm.

2) NMF training protocol: Practically, the estimation of $S_{M}$ is carried out during a short calibration phase. In particular, the user has to perform a simple specific motion: open and close his/her hand (two times). At the same time, the sEMG signal is acquired to build the matrix $E$ and then the NMF algorithm is applied for the computation of the muscular synergy matrix $S_{M}$ and the offline neural drives $U$.

3) Online ME proportional control of the grasp: The conceptual scheme of the online ME proportional control architecture is illustrated in Fig. 9. The user open/close his/her hand, de-facto controlling the fingers according to the first postural synergy joint pattern, as described in IV-B1. It follows that the human motor control system generates proper supraspinal neural drives to activate extensor and flexor groups of antagonistic muscles. These neural drives are not directly measurable. Therefore, we exploit the muscular synergy matrix $S_{M}$ to online estimate such neural drives from SEMG signals of the forearm muscles. Such online neural drives are used to control a virtual first postural synergy DoF, and to modulate the stiffness level of the grasp. The virtual synergistic DoF position reference is then mapped in the robotic hand joint space for the closure of each different grasp by means of specifics grasp postural mappings, as explained in Sect. III-B1.

In detail, once the muscular synergy matrix $S_{M}$ is estimated, it is possible to online compute the neural drives as

$$
U(t)=S_{M}^{+} E(t)
$$

where $U(t)=\left[u_{e}(t) u_{f}(t)\right]^{\mathrm{T}} \in \mathbb{R}^{2}$ is the vector of the instantaneous values of the neural drives, $S_{M}^{+}$is the pseudo-inverse matrix of $S_{M}$ and $E(t)=\left[e_{1}(t) \cdots e_{8}(t)\right]^{\mathrm{T}} \in \mathbb{R}^{8}$ is the vector of the instantaneous values of the sEMG channels. Two control signals for the robotic hand have to be derived from the estimated neural drives: the synergistic closure reference of the hand $\sigma^{r e f}(t)$, used in (4), and the grasp stiffness level $\xi(t)$, in (5). According to the antagonistic model concept given in Sect. IV-B1, these control signals are obtained by linear combination of the neural drive values as

$$
\begin{array}{ll}
\sigma^{r e f}(t) & =\frac{h_{1}}{2} u_{e}(t)-\frac{h_{2}}{2} u_{f}(t)+h_{3} \\
\xi(t) & =h_{4}\left(u_{e}(t)+u_{f}(t)\right)
\end{array}
$$

where $h_{1}, h_{2}, h_{3}$ and $h_{4}$ (note that $h_{3}=1$ ) are proper constants to normalize the neural drives values related to the flexor/extension actions and to scale $\sigma^{\text {ref }}$ and $\xi$ in the range $[0,1]$. Thus, the user has the possibility to control the joints configuration of the robotic device through free opening/closing movements of his/her hand and to regulate the grasp stiffness in order to vary the impedance of the grasp. Furthermore, a sound signal with a frequency proportional to the value of $\xi(t)$ is provided as an audio feedback to the user, so that a conscious online regulation of the stiffness level is possible.

\section{EXPERIMENTAL RESULTS}

To evaluate the usability of the HRI system proposed in this work, a series of grasping experiments has been conducted. In particular, four right handed and healthy male subjects, here indicated as S1, S2, S3 and S4, took part to the experiments.

The experimental session is composed by three phases. The first one is referred to as training session, where the trainsets for the ME control algorithms are collected, and a familiarization stage with a robotic hand graphical simulator is carried out. In the second and third phase the user attempts to grasp, both with the UB Hand IV and the gripper, a series of 9 objects that differ by shape, weight and rigidity (three objects for each type of grasp hand shape), listed in Tab. II.

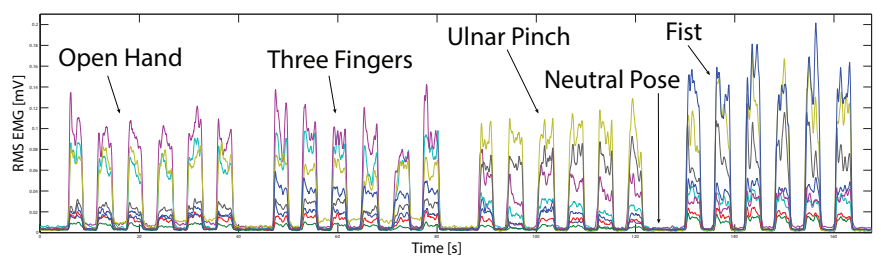

Fig. 10: Plot of the sEMG signals training dataset for subject S1. 

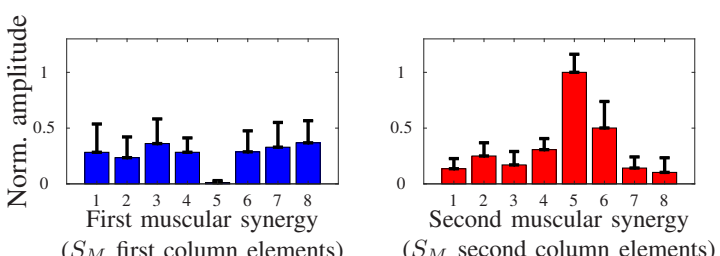

Fig. 11: Mean value of the muscular synergy weights for the four subjects.
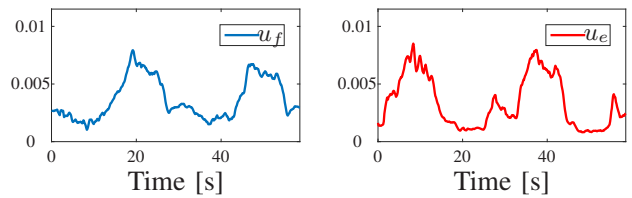

Fig. 12: Offline neural drives $u_{e}, u_{f}$ for subject S3.

\section{A. Training session}

First of all, the user has to autonomously place the electrodes on his forearm, see Sect. III-A. The placement of the electrodes is not completely repeatable and, since also the muscular structure varies among the users, the training session is intended to be user-dependent. After the electrode placement, the user performs the sequence of gestures during the recording of the eight sEMG channels for the training of the SVM classifier, following the indications of the graphical interface, Fig. 6. As an example, the training dataset acquired for subject S1 is reported in Fig. 10.

Once the SVM classifier has been built (see Sect. IV-A1), the second part of the training session dedicated to the NMFbased estimation of the muscular synergy matrix $S_{M}$ starts. During sEMG signals acquisition the user executes opening and closing movements as explained in Sect. IV-B2. The average synergy coefficients, averaged over the four subjects for the two columns of the muscular synergy matrix $S_{M}$, are reported in Fig. 11. The offline neural drives $u_{e}(t)$ and $u_{f}(t)$ estimated through the NMF algorithm for subject S3 are shown in Fig. 12, where it is possible to see in the graph on the left the neural drive responsible for the fingers flexions (blue), whereas on the right we can see the neural drive responsible for the extension actions (red).

When the muscular synergy matrix estimation is completed, the ME control system is ready to be used. At this point, the user controls the graphical simulator of the gripper, see Fig. 13, in order to familiarize with the HRI. In particular, a graphical reference named 'ghost gripper' is implemented in order to let the user follow, in simulation, a programmed closure and stiffness profile. This trial is composed of two phases regarding closure and stiffness tracking, and Fig. 14 shows results for the user S1: in Fig. 14(a), it is noticeable that the hand closure control signal modulated by the user (red) follows the desired tracking profiles (blue) of the ghost gripper, whereas, in Fig. 14(b), it can be observed how the subject successfully applies three requested levels of stiffness. Once the training/familiarization phase is performed, the recognition algorithm and the stiffness control do not require further tuning thanks to the robust setup and to the user-dependent training. Nevertheless, to increase robustness of the algorithms over multiple testing sessions, reinforcement learning techniques can be applied, as described in [47], [48], [49]. This will be part of future work.
TABLE II: Objects used in the experimental session.

\begin{tabular}{ccccc}
\hline $\begin{array}{c}\text { Object } \\
\text { type }\end{array}$ & $\begin{array}{c}\text { Size } \\
{[\mathbf{m m}]}\end{array}$ & $\begin{array}{c}\text { Weight } \\
\text { [g] }\end{array}$ & $\begin{array}{c}\text { UB Hand } \\
\text { grasp }\end{array}$ & $\begin{array}{c}\text { Gripper } \\
\text { grasp }\end{array}$ \\
\hline Bottle & $363 \times 96 \times 96$ & 400 & Power & Cylindrical \\
\hline Soft ball & $73 \times 73 \times 73$ & 180 & Tripodal & Tripodal \\
\hline Rigid ball & $73 \times 73 \times 73$ & 230 & Tripodal & Tripodal \\
\hline Small box & $88 \times 54 \times 40$ & 190 & Ulnar & Parallel \\
\hline Big box & $380 \times 200 \times 60$ & 500 & Ulnar & Parallel \\
\hline Paper cup & $114 \times 61 \times 61$ & 40 & Power & Cylindrical \\
\hline Adhesive tape & $84 \times 84 \times 50$ & 80 & Tripodal & Tripodal \\
\hline Spray can & $155 \times 45 \times 45$ & 255 & Power & Cylindrical \\
\hline Level & $420 \times 50 \times 20$ & 240 & Ulnar & Parallel \\
\hline
\end{tabular}

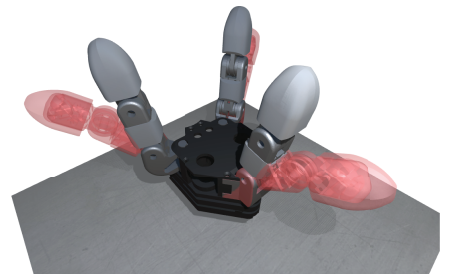

Fig. 13: Simulated gripper and ghost gripper (in red) for tracking experiments.

\section{B. Grasping task experiments}

During the grasping experiments, the subject is seated while looking at the nearby robotic hand. The experimenter hands out the objects one at a time, and reminds to the user about the correct grasp hand shape to choose. The grasp type associated to the object is previously decided, based on its physical form (see Tab. II). The overall grasp task is considered successful if the proper robotic hand shape is selected, and if the object is stably held (without squeezing it in case of a soft object) and then released, once it is asked, to the experimenter. For each subject, the objects to be grasped are 9, repeated 5 times in a random order for the UB Hand and for the gripper (avoiding the same grasp type for two consecutive objects), executing a total of 360 grasping tasks.

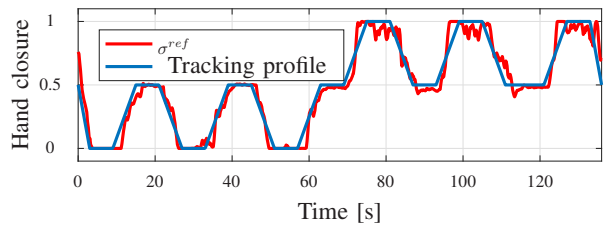

(a) Closure profile tracking.

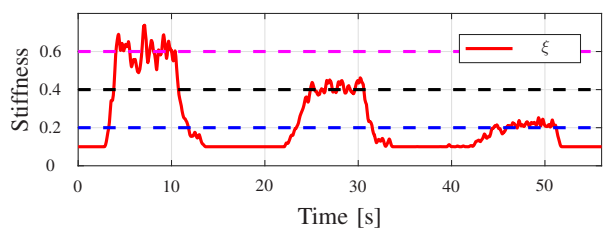

(b) Stiffness level tracking.

Fig. 14: Tracking for subject S1 in the training phase with the simulated gripper. 


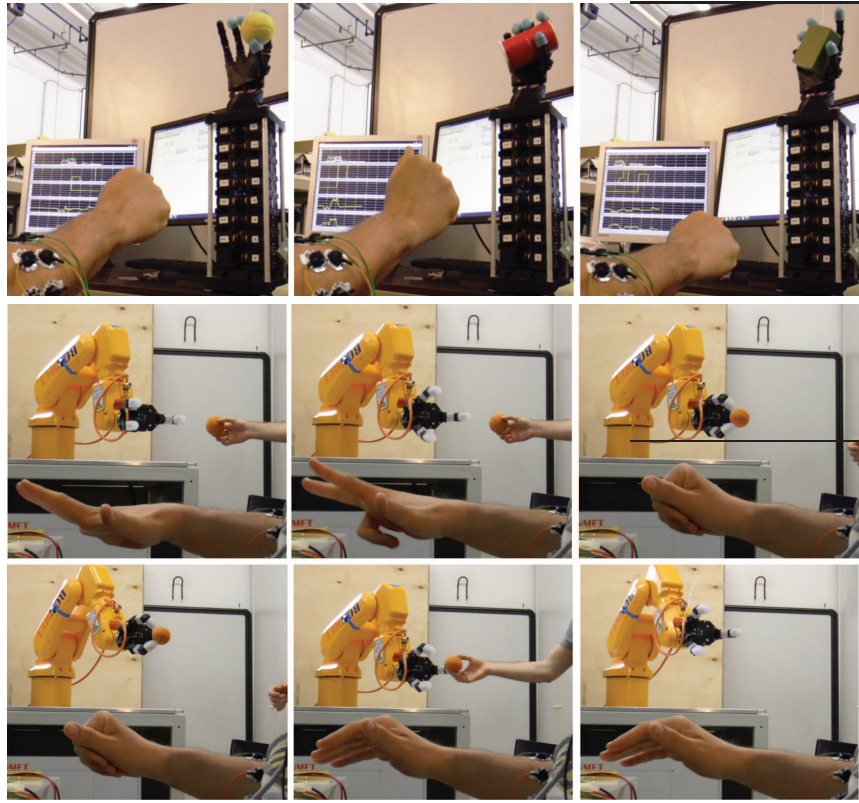

Fig. 15: Grasping tasks experiments with the UB Hand IV (top) and with the gripper (middle and bottom).

1) Object grasping with the UB Hand IV: The first part of the grasping tasks is executed using the anthropomorphic robotic hand. In the upper part of Fig. 15, it is possible to see three moments of this session, one for each type of grasp. In particular, we want to focus on the rate of success of: $(i)$ the selection of the desired grasp hand shape (i.e., the experimental accuracy of the SVM-based pattern recognition), and (ii) the overall grasp tasks (i.e., grab, hold and release the object). The success percentages computed over the five grasping series for each subject are reported in Tab. III.

2) Object grasping with the gripper: For these tests, the gripper is used as end-effector of a $6 \mathrm{DoF}$ industrial manipulator. The sequences of grasps are the same used with the UB Hand, but now the manipulator is programmed in order to automatically execute a sequence of movements. In details, the following motions are executed: (i) move to the workspace area where the object has to be grasped, (ii) lift and lower the held object, (iii) go to the release area and, finally, (iv) go back to the initial position. The subject has to perform the grasp (stage $(i) /(i i)$ ), stably hold the object (stages (ii)-(iii)) and release the object at the end of the task (stage (iii)/(iv)) according to the temporized steps. This sequence can be observed in Fig. 15. An interesting aspect in these experiments is the gripper/arm synchronization that must be achieved by the user, simulating an industrial-like situation with predetermined automatic sequences.

Fig. 16 depicts the recognized gestures for the selection of the grasp hand shape, the closure reference $\sigma^{\text {ref }}$ and the stiffness signal $\xi$, for the grasp of the objects Big Box, Paper Cup and Rigid Ball (Fig. 16(a), 16(c), 16(b) respectively ). The dashed lines delimit two parts of the graphs: one related to the grasp selection phase, and the other regarding the proportional control phase. On the left part it is possible to observe that the user executes an Open Hand, followed by an Ulnar Pinch to select the ulnar grasp for the robotic hand. Differently, on the right side the subject continuously controls the closure and stiffness of the chosen grasp, according to the object
TABLE III: Success percentages for the five grasping series.

\begin{tabular}{cccccc}
\hline $\begin{array}{c}\text { Evaluated } \\
\text { Task }\end{array}$ & $\begin{array}{c}\text { Success } \\
\text { for S1 }\end{array}$ & $\begin{array}{c}\text { Success } \\
\text { for S2 }\end{array}$ & $\begin{array}{c}\text { Success } \\
\text { for S3 }\end{array}$ & $\begin{array}{c}\text { Success } \\
\text { for S4 }\end{array}$ & $\begin{array}{c}\text { Average } \\
\text { Success }\end{array}$ \\
\hline G1 selection & $100 \%$ & $86.7 \%$ & $93.3 \%$ & $100 \%$ & $95 \%$ \\
\hline G2 selection & $93.3 \%$ & $100 \%$ & $93.3 \%$ & $80.7 \%$ & $91.8 \%$ \\
\hline G3 selection & $100 \%$ & $93.3 \%$ & $100 \%$ & $93.3 \%$ & $96.7 \%$ \\
\hline Grasp completion & $100 \%$ & $97.5 \%$ & $95 \%$ & $92.5 \%$ & $96.3 \%$ \\
\hline
\end{tabular}

characteristics. It is possible to see how the stiffness value is naturally adjusted: a higher level is used to grasp the Big Box, that presents greater dimensions and weight, whereas a lower level is necessary to grasp the lighter, but not flexible, Rigid Ball, and an almost minimum value is provided to not squeeze the very soft Paper Cup. Note also that, in the latter case, the synergistic closure reference is not brought to the maximum limit by the user, in order to act a more delicate grasp action. Similar behaviors have been performed by all the subjects in a totally independent and natural manner, once the training and familiarization phases were carried out.
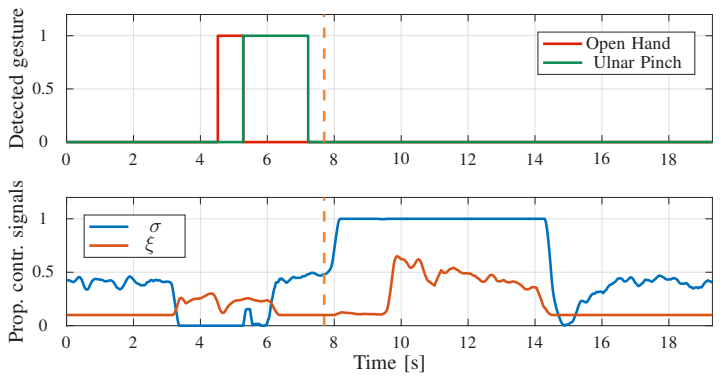

(a) Big Box

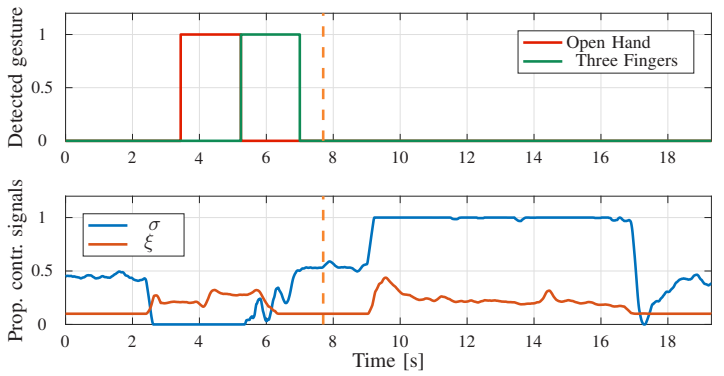

(b) Rigid Ball
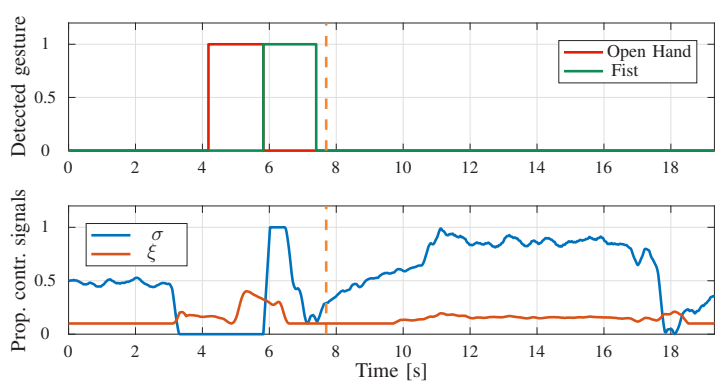

(c) Paper Cup

Fig. 16: Temporal plot of the control signals for the grasp of the objects Big Box, Rigid Ball and Paper Cup. 


\section{CONCLUSION}

The improvement of flexibility and functionality of robotic grasping systems emulating human capabilities is a fundamental aspect for the widespread acceptance of HRIs. To improve the integration between subject and robot, we have designed an embedded wearable interface based on a control system that exploits neuromuscular information to allow the user regulating in a natural fashion the behavior of an artificial hand during grasping tasks. Leveraging the combination of ML-based pattern recognition and proportional control of the grasp closure and stiffness, the motor control of the human hand has been emulated, since the muscular activation patterns are related with the intended movements while the agonistantagonist dynamics is responsible of the regulation of the grasp impedance. The human-like degrees of control provided to the user of the HRI system combined with the possibility of selecting the grasp shape has been tested through several grasping experiments on a dexterous anthropomorphic robotic hand and on an industrial gripper mounted on a manipulator, resulting in a mean success ratio of $96.3 \%$ among 4 healthy subjects.

Our future work will focus on several aspects. The improvement of the HRI system performance from a user-centred point of view will be studied in depth, first of all investigating a more effective feedback information modality to the subject, preferably based on an haptic feedback interface. The possibility to extract information about more than one postural synergy from muscle activations will be explored, taking into account the possibility of using novel combination of ML- and factorization-based techniques and analyzing the EMG activity from both extrinsic and intrinsic human hand's muscles. Finally, we will focus on the optimization of the embedded solution, exploiting sensor fusion techniques to obtain a more natural control of grasps and movements of the robotic arm.

\section{REFERENCES}

[1] J. L. Collinger et al., "High-performance neuroprosthetic control by an individual with tetraplegia," The Lancet, 2013.

[2] J. Meng et al., "Noninvasive electroencephalogram based control of a robotic arm for reach and grasp tasks," Scientific Reports, vol. 6, 2016.

[3] S. Benatti et al., "Scalable eeg seizure detection on an ultra low power multi-core architecture," in BIOCAS 2016. IEEE, 2016.

[4] N. V. Thakor, "Translating the brain-machine interface," Science translational medicine, vol. 5, no. 210, pp. 210ps17-210ps17, 2013.

[5] D. Atkins et al., "Epidemiologic overview of individuals with upper-limb loss and their reported research priorities." JPO: Journal of Prosthetics and Orthotics, vol. 8, no. 1, pp. 2-11, 1996.

[6] S. Benatti et al., "A prosthetic hand body area controller based on efficient pattern recognition control strategies," Sensors, 2017.

[7] D. Farina et al., "Human? machine interfacing by decoding the surface electromyogram," IEEE Signal Processing Magazine, 2015.

[8] M. A. Oskoei and H. Hu, "Myoelectric control systemsa survey," Biomedical Signal Processing and Control, 2007.

[9] D. Novak and R. Riener, "A survey of sensor fusion methods in wearable robotics," Robotics and Autonomous Systems, 2015.

[10] D. Farina et al., "The extraction of neural information from the surface emg for the control of upper-limb prostheses: emerging avenues and challenges," IEEE TNSRE, 2014.

[11] B. Peerdeman et al., "Myoelectric forearm prostheses: State of the art from a user-centered perspective," USDVA, 2011.

[12] P. K. Artemiadis et al., "A switching regime model for the emgbased control of a robot arm," IEEE Transactions on SMC, Part B (Cybernetics), 2011

[13] K. Kiguchi et al., "An emg-based control for an upper-limb power-assist exoskeleton robot," IEEE Transactions on SMC, Part B, 2012.

[14] L. A. Jones and S. J. Lederman, Human hand function. Oxford University Press, 2006.

[15] M. Santello et al., "Postural hand synergies for tool use," The Journal of Neuroscience, 1998.

[16] S. Marco et al., "Hand synergies integration of robotics and neuroscience for understanding the control of biological and artificial hands," Physics of life reviews, 2016.
[17] E. Bizzi et al., "Computations underlying the execution of movement: a biological perspective," Science, 1991.

[18] A. d'Avella et al., "Control of fast-reaching movements by muscle synergy combinations," The Journal of neuroscience, vol. 26, 2006.

[19] L. H. Ting et al., "Neuromechanics of muscle synergies for posture and movement," Current opinion in neurobiology, 2007.

[20] R. Osu et al., "Multijoint muscle regulation mechanisms examined by measured human arm stiffness and emg signals," Journal of neurophysiology, 1999.

[21] N. Hogan, "Controlling impedance at the man/machine interface," in Robotics and automation, 1989. Proceedings., 1989 IEEE international conference on. IEEE, 1989, pp. 1626-1631.

[22] C. Melchiorri et al., "Development of the ub hand iv: Overview of design solutions and enabling technologies," IEEE Robotics \& Automation Magazine, 2013.

[23] G. Palli et al., "The dexmart hand: Mechatronic design and experimental evaluation of synergy-based control for human-like grasping," The International Journal of Robotics Research, 2014.

[24] (2016) Ottobock. [Online]. Available: http://www.ottobockus.com/

[25] (2016) Bebionic web site. [Online]. Available: http://www.bebionic.com/

[26] (2016) Touchbionics web site. [Online]. Available: http://www.touchbionics.com/

[27] D. S. Naidu et al., "Control strategies for smart prosthetic hand technology: An overview," in EMBC 2008. IEEE, 2008.

[28] S. A. a. o. Dalley, "A method for the control of multigrasp myoelectric prosthetic hands," IEEE TNSRE, 2012.

[29] F. V. Tenore et al., "Decoding of individuated finger movements using surface electromyography," IEEE Transactions on Biomedical Engineering, vol. 56, no. 5, pp. 1427-1434, 2009.

[30] E. Scheme et al., "Electromyogram pattern recognition for control of powered upper-limb prostheses: State of the art and challenges for clinical use," Journal of rehabilitation research and development, vol. 48, no. 6, p. 643, 2011.

[31] L. J. Hargrove et al. " "Multiple binary classifications via linear discriminant analysis for improved controllability of a powered prosthesis," IEEE TNSRE, vol. 18, no. 1, pp. 49-57, 2010.

[32] N. Fligge, H. Urbanek, and P. van der Smagt, "Relation between object properties and emg during reaching to grasp," Journal of Electromyography and Kinesiology, vol. 23, no. 2, pp. 402-410, 2013.

[33] E. Biddiss and T. Chau, "Upper-limb prosthetics: critical factors in device abandonment," American journal of physical medicine \& rehabilitation, vol. 86, no. 12, pp. 977-987, 2007.

[34] N. Jiang et al., "Extracting simultaneous and proportional neural control information for multiple-dof prostheses from the surface electromyographic signal," IEEE Transactions on Biomedical Engineering, 2009.

[35] S. Benatti et al., "Multiple biopotentials acquisition system for wearable applications," in BIODEVICES. Scitepress, 2015.

[36] P. Schnle et al., "A dc-connectable multi-channel biomedical data acquisition asic with mains frequency cancellation," in ESSCIRC (ESSCIRC), 2013 Proceedings of the, 2013.

[37] A. Perotto and E. F. Delagi, Anatomical guide for the electromyographer: the limbs and trunk. Charles C Thomas Publisher, 2005.

[38] C. J. De Luca et al., "Filtering the surface emg signal: Movement artifact and baseline noise contamination," Journal of biomechanics, 2010.

[39] S. Benatti et al., "A sub-10mw real-time implementation for emg hand gesture recognition based on a multi-core biomedical soc," in IWASI 2017. IEEE, 2017.

[40] (2016) Robotis corp. web site. [Online]. Available: http://www.en.robotis.com/

[41] F. Ficuciello et al., "Experimental evaluation of postural synergies during reach to grasp with the ub hand iv," in 2011 IEEE/RSJ. IEEE, 2011.

[42] C. J. Burges, "A tutorial on support vector machines for pattern recognition," Data mining and knowledge discovery, 1998.

[43] H. Wu et al., "Classification of solder joint using feature selection based on Bayes and support vector machine," IEEE Transactions on Components, Packaging and Manufacturing Technology, 2013.

[44] C.-C. Chang and C.-J. Lin, "Libsvm: a library for support vector machines," ACM TIST, 2011.

[45] M. W. Berry et al., "Algorithms and applications for approximate nonnegative matrix factorization," Computational statistics \& data analysis, 2007.

[46] E. Burdet et al., "Interaction force, impedance and trajectory adaptation: by humans, for robots," in Experimental Robotics. Springer, 2014.

[47] A. H. Oskouei et al., "Intra-session and inter-day reliability of forearm surface emg during varying hand grip forces," Journal of Electromyography and Kinesiology, 2013.

[48] R. C. Araujo et al., "On the inter-and intra-subject variability of the electromyographic signal in isometric contractions," Electromyography and clinical neurophysiology, 2000.

[49] D. A. Gabriel, "Changes in kinematic and emg variability while practicing a maximal performance task," Journal of Electromyography and Kinesiology, 2002. 